\title{
Clinical Presentation and Risk Factors for Orbital Infections in a Tertiary Hospital
}

\author{
Olusola Joseph Omotoye*, Iyiade Adeseye Ajayi, Olanike Folashade Bodunde
}

Department of Ophthalmology, Ekiti State University Teaching Hospital, Ado Ekiti, Nigeria

\section{Email address:}

layoshol@yahoo.com (O. J. Omotoye)

${ }^{*}$ Corresponding author

\section{To cite this article:}

Olusola Joseph Omotoye, Iyiade Adeseye Ajayi, Olanike Folashade Bodunde. Clinical Presentation and Risk Factors for Orbital Infections in a Tertiary Hospital. International Journal of Infectious Diseases and Therapy. Vol. 5, No. 3, 2020, pp. 51-55.

doi: $10.11648 /$ j.ijidt.20200503.13

Received: June 26, 2020; Accepted: July 15, 2020; Published: July 23, 2020

\begin{abstract}
Background: Orbital infections are common ophthalmic emergencies with sight and life threatening if not promptly and adequately managed. Purpose: To determine the incidence of orbital infections and the risk factors that predispose these patients to orbital infections in our tertiary health institution. Methods: Records of all patients with orbital infections who presented at the ophthalmic plastic clinic between January 2010 and December 2019 were retrieved. Demographic characteristics, duration of symptoms, presenting visual acuity, and oculoplastic procedures were obtained. Results: Sixty six patients presented with orbital infections which constituted (4.5\%) of all ocular emergencies seen in this centre. There were $44(66.7 \%)$ males and $22(33.3 \%)$ females. Preseptal cellulitis was $49(74.2 \%)$ while orbital cellulitis was $17(25.8 \%)$. More than $9 / 10^{\text {th }}$ of preseptal cellulitis were found in children of 5 years and below. The proportion of visual impairment and blindness was higher in orbital cellulitis than preseptal cellulitis. The most common risk factors for preseptal cellulitis was ocular allergy while sinus infection was commonest in orbital cellulitis. All patients with orbital cellulitis were admitted into the eye wards out of which $2(11.8 \%)$ of these patients died of septiceamia. Conclusion: Orbital infection is a relatively common ophthalmic emergency which is a cause of visual impairment and blindness. The most common risk factor for preseptal cellulitis was ocular allergy while sinus infection was commonest in orbital cellulitis. Aggressive and goal oriented awareness campaign for early presentation of patients with these infections at eye care facility is advocated to prevent unnecessary death from this ophthalmic emergency.
\end{abstract}

Keywords: Cellulitis, Emergency, Hospital, Infections, Orbital

\section{Introduction}

Orbital infections are common infections of ocular adnexia and orbital tissues. [1] The infection posterior to the orbital septum is called orbital cellulitis while the anterior ones are called pre-septal cellulitis. Sometimes the infections can be a continuum. [2] Pre-septal cellulitis is reported to be more prevalent than post-septal infection in children below the age of 5yrs. [3] Orbital infections develop via direct inoculation of infectious materials to the orbital soft tissue secondary to trauma, surgery or orbital foreign bodies; extension from adjacent structures and heamatogenous spread (seedings secondary to sepsis or bacteria endocarditis). As much as $60 \%$ of these orbital infections result from direct spread of sinusitis, with the ethmoidal being the most commonly implicated, owing to its thin and porous wall. Spread can also be from pre-septal spaces, pharynx, middle ear, facial skin, nose, lacrimal gland (dacrocystitis) or dentition from an external ocular infection, or following trauma to the eyelids. However, primary sources of infection can be local skin trauma, sinus infections or penetrating trauma, specifically those involving the ethmoid sinus [4] Patients with preseptal or orbital cellulitis present with similar symptoms, such as eye pain, periorbital swelling, and/or fever. [4] Prompt administration of effective antibiotics has resulted in a dramatic reduction of complication associated with those infections. [2, 5] They can cause significant local and systemic morbidity and more than $10 \%$ of patients suffer loss of vision in the eye. [6] It is therefore an ophthalmic emergency with sight threatening complications of visual 
loss. The severe form can be life threatening if not promptly and adequately managed. [5]

This study is aimed at determining the incidence of orbital infections and the risk factors that predispose these patients to orbital infections in our tertiary health institution. This is to reduce the life threatening complications that could arise from this ophthalmic emergency.

\section{Materials and Methods}

Records of all patients with orbital infections of all age groups who presented at the ophthalmic plastic clinic between January 2010 and December 2019 were retrieved. All patients were examined following referral from ophthalmologists from other subspecialty (Retina, Glaucoma and general ophthalmology clinics) after which clinical diagnoses were made. Demographic characteristics, duration of symptoms, presenting visual acuity, oculoplastic procedures were obtained from the records. Visual acuity were categorized with $\geq 6 / 18$ as normal, $<6 / 18$ to $>3 / 60$ as visual impairment and $<3 / 60$ as blindness. [7] Infants were classified as either blind or believed not to be blind. Ethical approval was obtained from the institution's ethical review committee though data collection did not directly involve patient's participation. This study was carried out in line with the ethical standards according to the Helsinki Declaration of 1975 as revised in 1983. Data obtained were recorded and analyzed using Statistical Package for Social Sciences (SPSS) version 25. Means (Standard deviations) were used to describe the distributions of continuous variables. Categorical variables were described in Percentages. Comparisons of categorical data was performed with the use of Pearson's chi-square test. $P<0.05$ was considered statistically significant.

\section{Results}

Sixty six patients presented with features of orbital infections during this period. This constituted (4.54\%) of all ocular emergencies $(1,480)$ seen in this centre. There were 44 $(66.7 \%)$ males and $22(33.3 \%)$ females with a male to female ratio of $2: 1$. The ages ranged from 3 months to 75 years with mean age of $20.76 \pm 22.35$ years with $23(51.1 \%)$. Unilateral cases occurred in $60(90.9 \%)$ with 33 cases occurring in the right eyes and 27 in the left eyes while bilateral was $6(9.1 \%)$ making a total of 72 eyes. $(\chi 2=18.273, \mathrm{df}=2, \mathrm{p}=0.001)$.

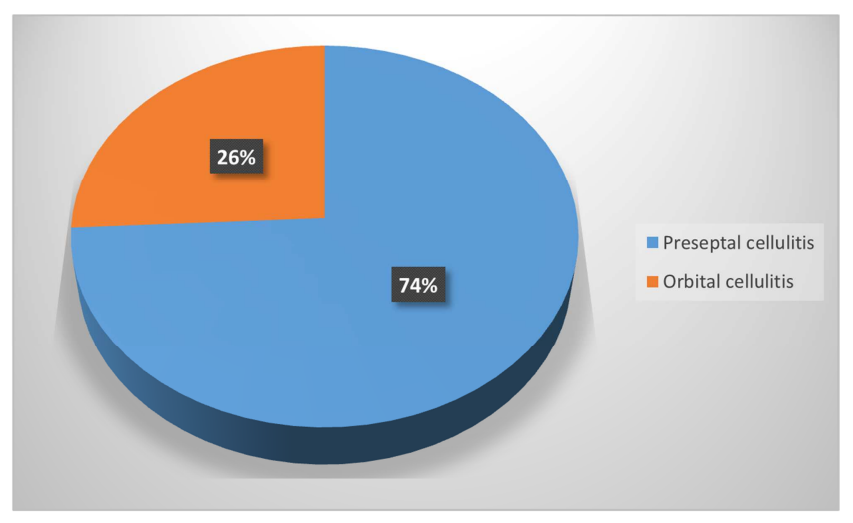

Figure 1. Orbital Infections in Oculoplastic Clinic.

Preseptal cellulitis was $49(74.2 \%)$ while orbital cellulitis was $17(25.8 \%)(\chi 2=15.515, \mathrm{df}=2, \mathrm{p}=0.001)$.

Table 1. Age and sex distribution.

\begin{tabular}{lll}
\hline Age group (yrs) & male n (\%) & female n (\%) \\
\hline $0-10$ & $25(78.1)$ & $7(21.9)$ \\
$11-20$ & $5(45.5)$ & $6(54.5)$ \\
$21-30$ & $3(75.0)$ & $1(25.0)$ \\
$31-40$ & $4(66.7)$ & $2(33.3)$ \\
$41-50$ & $3(75.0)$ & $1(25.0)$ \\
$51-60$ & $1(50.0)$ & $1(50.0)$ \\
$>60$ & $3(42.9)$ & $4(57.1)$ \\
Total & $44(66.7)$ & $22(33.3)$ \\
\hline
\end{tabular}

Preseptal cellulitis had a higher proportion than orbital cellulitis in all age group except in patients greater than 50 years age group.

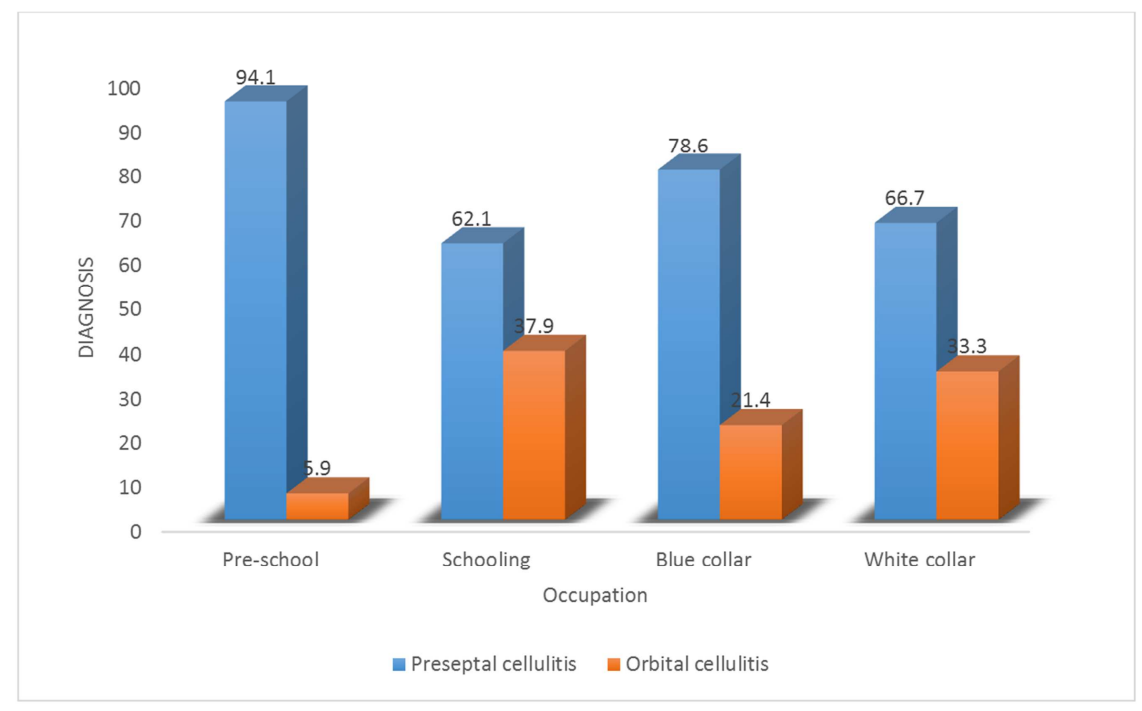

Figure 2. Occupational classification of patients with orbital infections. 
The proportion of preseptal cellulitis is higher in all the occupational groups than orbital cellulitis however, orbital cellulitis had the highest proportion in the schooling age- group.

Table 2. Presentation of Patients with Orbital Infections.

\begin{tabular}{lll}
\hline \multirow{2}{*}{ Variables } & Diagnosis & Orbital cellulitis n (\%) \\
\cline { 2 - 3 } & Preseptal cellulitis n (\%) & \\
Age class (yrs) & & $1(4.5)$ \\
>5yrs & $21(95.5)$ & $16(36.4)$ \\
Visual acuity & $28(63.6)$ & \\
Normal vision & & $5(10.9)$ \\
Visual impairment & $41(89.1)$ & $5(62.5)$ \\
Blindness & $3(37.5)$ & $7(58.3)$ \\
Risk factors & $5(41.7)$ & \\
Ocular Allergy & & $1(5.6)$ \\
Ear infection & $17((94.4)$ & $1(50.0)$ \\
Facial infection & $1(50.0)$ & $2(33.3)$ \\
Sinus infection & $4(66.7)$ & $9(45.0)$ \\
Trauma & $11(55.0)$ & $2(25.0)$ \\
$*$ URTI & $6(75.0)$ & $2(16.7)$ \\
\hline
\end{tabular}

*URTI-upper respiratory tract infections.

More than $9 / 10^{\text {th }}$ of preseptal cellulitis 21 (95.5\%) were found in children of 5 years and below compared to orbital cellulitis $1(4.5 \%)$. The proportion of visual impairment and blindness was higher in orbital cellulitis than preseptal cellulitis. The most common risk factors for preseptal cellulitis was ocular allergy while sinus infection was commonest in orbital cellulitis. None of these patients had systemic morbidities like Diabetes mellitus or Renal pathology.

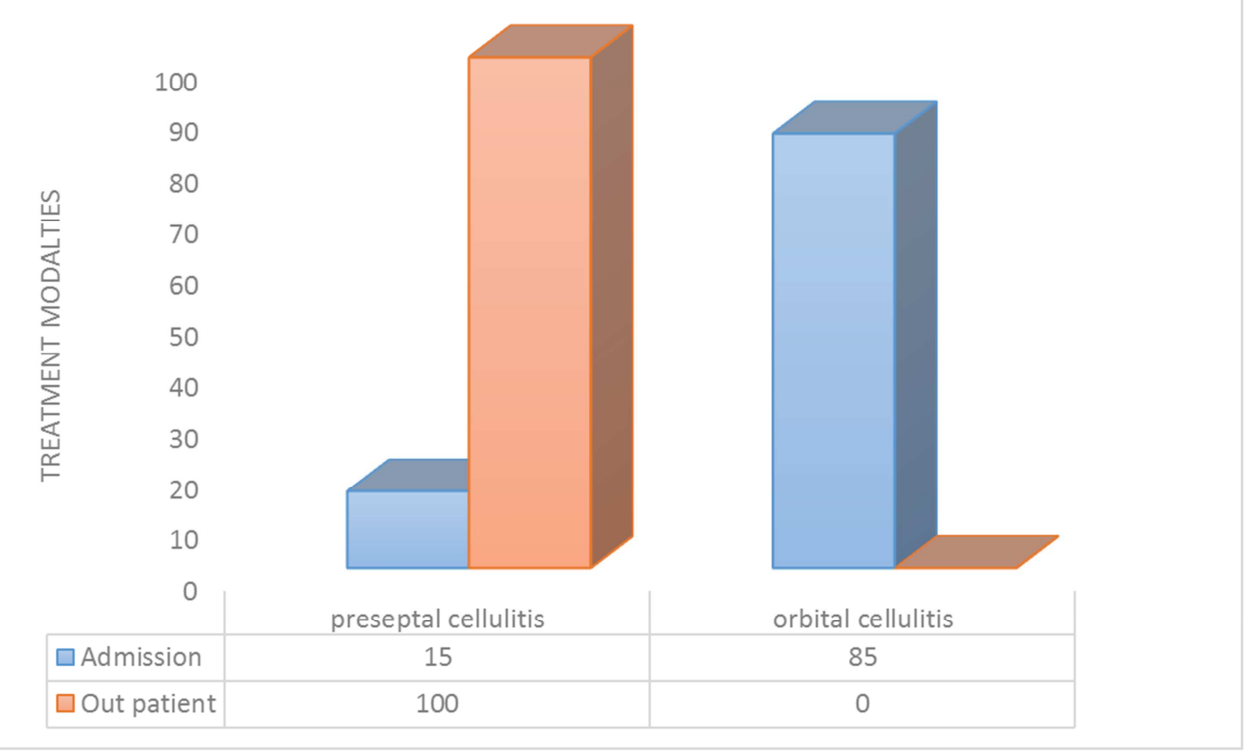

Figure 3. Treatment modalities of patients with orbital infections.

Of forty nine patients with preseptal cellulitis, 46 (93.4\%) were managed on out- patient basis while all patients with orbital cellulitis were admitted into the eye wards. These patients with orbital cellulitis were placed on both topical and systemic broad spectrum antibiotics empirically. Out of these, $2(11.8 \%)$ patients died of severe complications of Septiceamia.

\section{Discussion}

Orbital infection is one of the relatively common ophthalmic emergencies which constituted about $4 \%$ of all ocular emergencies seen in this centre. These ophthalmic emergencies require urgent medical attention in order to avert high risk ocular complications which can lead to permanent visual loss. Even when vision loss or blindness do not occur, cosmetic blemish like phthisical eye may occur if prompt and appropriate actions are not taken. [8] The incidence of orbital infections in this centre is comparable with others in the literature $[1,5,9-10]$ though most only reported incidence of either pre-or post-septal orbital infections. It has been reported that preseptal cellulitis is more common [11] but less aggressive than orbital cellulitis. [9] Most of these 
infections in this centre occurred in the first decade of life with male preponderance as reported in many studies. $[5,13]$ However, a southwestern study in Nigeria reported a female preponderance. [12] The trend in this study is in keeping with serious childhood infections occurring in males. [6] Therefore, attending physician should intervene as quickly as these children with features suggestive of orbital infections present in the health facility. This is to avert clinical deterioration that may be associated with early and subtle post-septal clinical features. [6] Orbital cellulitis in adults over 50 years can aggressively lead to intracranial extension resulting in meningitis, abscess or carvenous sinus thrombosis which are severe manifestations of orbital infection which if neglected could lead to loss of life. [6]

This study revealed that preseptal orbital infections was commonest in the preschool age group while orbital cellulitis was significantly high in the schooling and white collar job. Early presentation of Orbital infections in a preschool age group in a health facility is dependent on the care- giver which in turn is influenced by some factors such as cost, lack of awareness, cultural beliefs and personal factors which could act as barriers to eye care facility utilization [14]. All these factors might lead to delay in presentation at the eye care facility. A further delay in managing these patients due hospital bureaucracy might result in either prolonged hospital stay or avoidable death of the patients.

More than $9 / 10^{\text {th }}$ of preseptal cellulitis were found in children of 5 years and below compared to orbital cellulitis. This is similar to many other studies. [6, 11-12] These children should be managed and monitored closely to forestall progression of preseptal cellulitis to more aggressive orbital cellulitis as reported by Lim et al in their study. [15] The proportion of visual impairment and blindness was higher in orbital cellulitis than preseptal cellulitis. This is in keeping with the fact that orbital cellulitis is more sightthreatening than preseptal cellulitis thus every effort should be geared towards reduction of the sequale of visual morbidity from this ophthalmic emergencies.

The most common risk factors for preseptal cellulitis was ocular allergy followed by sinus infections which was closely followed by upper respiratory tract infections while in orbital cellulitis, sinus infection was the commonest associated risk factors occurring in more than fifty percent of the patients. This was similar to many other studies. [4, 16] These associated risk factors should be identified early and properly managed to avert a very serious ocular morbidity and mortality. Some of these patients were co-managed with the Ear -Nose and Throat Surgeon who performed Bilateral Antral wash out (BAWO) under antibiotics cover after which the patients that recovered well were discharged home. All the patients that had orbital cellulitis were admitted into the ward and managed appropriately. Some of them had surgical procedures like BAWO by the Ear -Nose and Throat (ENT) Surgeon and Incision and Drainage (I\&D) were done by the ophthalmologist. Two (11.8\%) of the patients with severe form of orbital cellulitis died of septiceamia due to late presentations in the eye facility. This is slightly higher than the report from Goncalves et al study. [17] This could have been prevented by health education on early presentation to health facility. An aggressive health education in Ekiti community to prevent delay in presentation after having an eye emergency was advocated in previous publication. [8] Other barriers to accessing eye care like cost, distance to the existing eye care facility, ignorance or poor knowledge on the use of unorthodox eye medication practice [7] should also be addressed to avert the unnecessary death.

\section{Conclusion}

Orbital infections is a relatively common ophthalmic emergency with Preseptal orbital infections occurring predominantly in the pre-school age group while orbital cellulitis was significantly high in the schooling and white collar job patients. It is a cause of visual impairment and blindness. The most common risk factors for preseptal cellulitis was ocular allergy while sinus infection was commonest in orbital cellulitis. Aggressive and goal oriented awareness campaign for early presentation of patients with these infections at eye care facility is advocated to prevent unnecessary death from this ophthalmic emergency.

\section{Abbreviations}

1. SPSS- Statistical Package for Social Sciences

2. URTI- Upper respiratory tract infections

3. BAWO-Bilateral Antral wash out

\section{Declarations}

\section{Ethics Approval and Consent to Participate}

Ethical approval was obtained.

\section{Consent for Publication}

Not Applicable.

\section{Availability of Data and Materials}

The datasets used and/or analysed during the current study are available from the corresponding author on reasonable request.

\section{Competing Interest}

The authors declare that we have no competing interest.

\section{Funding}

No money grant was received.

\section{Authors Contributions}

OJ OMOTOYE: concept, design, drafting and final approval IA AJAYI: design, drafting, critical analysis and final approval

OF BODUNDE: design, drafting and final approval 


\section{Acknowledgements}

Not applicable.

\section{References}

[1] Shoaei SD, Tehrani S, Arab-Mazar Z. Frequency of Preseptal Cellulitis and Its Risk Factors in Patients Admitted to Two Educational Hospitals in Tehran, Iran, During 2014-2015. International Journal of Infection. 2017; 4 (2).

[2] Harrington JN. Orbital cellulitis. 2016.

[3] Mbbs MF, Fraco AM. Current treatment and outcome in orbital cellulitis. Australian and New Zealand journal of ophthalmology. 1999; 27 (6): 375-379.

[4] Carlisle RT, Fredrick GT. Preseptal and orbital cellulitis. Hosp Physician. 2006; 42 (10): 15-19.

[5] Uhumwangho OM, Kayoma DH. Current trends in treatment outcomes of orbital cellulitis in a tertiary hospital in Southern Nigeria. Nigerian Journal of Surgery. 2016; 22 (2): 107-110.

[6] Hamed-Azzam S, AlHashash I, Briscoe D, Rose GE, Verity DH. Common orbital infections $\sim$ state of the art part I. Journal of ophthalmic \& vision research. 2018; 13 (2): 175.

[7] WHO: Categories of visual impairment: Strategies for the prevention of blindness in National programmes. Technical report series, No 10 Geneva 1984.

[8] Joseph OO, Adeseye AI. Profile of ocular emergencies in a tertiary health centre. IOSR Journal of Dental and Medical Sciences. 2016; 15 (07): 75-79.
[9] Babar TF, Zaman M, Khan MN, Khan MD. Risk factors of preseptal and orbital cellulitis. J Coll Physicians Surg Pak. 2009; 19 (1): 39-42.

[10] Georgakopoulos CD, Eliopoulou MI, Stasinos S, Exarchou A, Pharmakakis N, Varvarigou A. Periorbital and orbital cellulitis: a 10-year review of hospitalized children. European journal of ophthalmology. 2010; 20 (6): 1066-1072.

[11] Ambati BK, Ambati J, Azar N, Stratton L, Schmidt EV. Periorbital and orbital cellulitis before and after the advent of Haemophilus influenzae type B vaccination. Ophthalmology. 2000; 107 (8): 1450-1453.

[12] Onakpoya OH, Adeoye AO, Akinpelu OV. Cost-Related Antibiotic Dosage Omissions-Challenge for Orbital Cellulitis Management in Resource Poor Communities. Orbit. 2009; 28 (2-3): 147-152.

[13] Nageswaran S, Woods CR, Benjamin Jr DK, Givner LB, Shetty AK. Orbital cellulitis in children. The Pediatric infectious disease journal. 2006; 25 (8): 695-699.

[14] Ebeigbe Ja, Ovenseri-Ogbomo Go. Barriers To Utilization Of Eye Care Services In Rural Communities In Edo State, Nigeria. Borno Medical Journal 2014; 11 (2) 98-104.

[15] Lim L, Miller D, Ah-Kee E, Ferguson A. Preseptal cellulitis or orbital cellulitis. West Indian Med J. 2015; 65 (1).

[16] Chaudhry I, Shamsi F, Arfaj K, et al. Predisposing Factors for Preseptal Cellulitis Among Patients Presenting to a Tertiary Eye Care Center. Investigative Ophthalmology \& Visual Science. 2007; 48 (13): 347-347.

[17] Gonçalves R, Menezes C, Machado R, Ribeiro I, Lemos JA. Periorbital cellulitis in children: analysis of outcome of intravenous antibiotic therapy. Orbit. 2016; 35 (4): 175-180. 